ESAIM: PROCEEDINGS, April 2007, Vol.17, 50-66

Alain Piétrus \& Michel H. Geoffroy, Editors

\title{
ON THE CONTROL OF ILL-POSED DISTRIBUTED PARAMETER SYSTEMS
}

\author{
R. Dorville ${ }^{1}$, O. Nakoulima ${ }^{2}$ And A. OMrane $^{3}$
}

\begin{abstract}
We show that the so-called low-regret (or least-regret) control by J. L. Lions [8] fits on the control of ill-posed problems. At each time, we give the characterization of the so-called no-regret control by means of singular optimality systems. For the backward heat ill-posed problem, no Slater hypothesis is assumed on the admissible set of controls $\mathcal{U}_{\text {ad }}$.

This work is two pieces, and two methods are considered : the regularization method and the nullcontrollability method. For the first method, a zero order corrector is used, while for the second method, the passage to the limit is easy. The results presented here generalize the works in $[2,3]$ to the no-regret control.
\end{abstract}

Résumé. On montre que le contrôle à moindres regrets de J. L. Lions [8] est bien adapté pour le contrôle des des problèmes mal posés. A chaque fois, on donne une caractérisation du contrôle sans regret par le moyen de systèmes d'optimalité singuliers. Pour le problème de chaleur rétrograde mal posé, aucune hypothèse de type Slater sur l'ensemble des contrôles admissibles $\mathcal{U}_{\text {ad }}$ n'est nécessaire. Ce travail est divisé en deux parties, et deux méthodes sont considérées: la méthode de régularisation et celle de la contrôlabilité à zéro. Pour la première méthode, un correcteur d'ordre zéro est utilisé, alors que pour la seconde méthode le passage à la limite est simple. Les résultats présentés ici généralisent les travaux dans $[2,3]$ au contrôle sans regret.

\section{Contents}

1. Introduction $\quad 51$

1.1. Preliminaries $\quad 51$

1.2. Existence of a solution for the ill-posed heat problem 52

1.3. The optimal control question $\quad 53$

2. The regularization approach $\quad 54$

2.1. A priori estimates. The low-regret control 57

2.2. Zero-order corrector $\quad 59$

2.3. Passage to the limit 61

3. The controllability approach 62

3.1. The low-regret control for the null-controllable problem 63

3.2. Passage to the limit $\quad 65$

$\begin{array}{ll}\text { References } & 66\end{array}$

\footnotetext{
${ }^{1}$ Université Antilles-Guyane, Laboratoire d'Analyse, Optimisation et Contrôle (AOC), UFR Sciences Exactes et Naturelles, Campus de Fouillole, 97159 Pointe à Pitre. E-mail: rene.dor@wanadoo.fr

2 onakouli@univ-ag.fr

3 aomrane@univ-ag.fr
} 


\section{IntRoduction}

The solutions of singular optimal control problems, particularly of ill-posed problems are not regular. As a consequence, the characterization of such solutions has not been deeply investigated. Moreover, the assumptions on the control problem are frequently chosen in such a way that some standard methods can be applied to derive the existence of solutions.

We consider here the prototype of ill-posed problems : the ill-posed backward heat problem, with controls $v$ in a non-empty closed convex subset $\mathcal{U}_{\text {ad }}$ of the Hilbert space $L^{2}$, and a cost functional.

It is well known that if we add the Slater type hypothesis as $: \mathcal{U}_{\text {ad }}$ has a non-empty interior, then we ensure the existence of an optimal control, using standard methods (see Lions [6]). But, we do not know if this hypothesis is unavoidable!

One way to deal with this problem -instead of answering the question-, is to propose another approach, where the Slater hypothesis on $\mathcal{U}_{\text {ad }}$ is not needed. Convex cones as $\left(L^{2}\right)^{+}$which are empty interiored may be used as set of feasible controls $v$. We here use the regularization approach in a first part, and the null-controllability approach in a second part. In both methods, a new datum is introduced. These data are supposed to be chosen as largely as possible, say in a vector space. That is the data are incomplete, and in this case, the standard methods as the penalization method are not adapted (see Lions [8], and Nakoulima-Omrane-Velin [13] [14]). We then seek for the low-regret control of the distributed system of incomplete data obtained. And thus, the no-regret control of the original problem appears naturally by taking some limit, which is possible without the Slater hypothesis.

Roughly speaking, the low-regret control $u^{\gamma}$ satisfies to the following inequality :

$$
J\left(u^{\gamma}, g\right) \leq J(0, g)+\gamma\|g\|_{Y}^{2} \quad \forall g \text { in a Hilbert space } Y,
$$

where $\gamma$ is a small positive parameter ( $g$ being the pollution or incomplete data). With the low-regret control we admit the possibility of making a choice of controls $v$ 'slightly worse' than by doing better than $v=0$-but 'not much' if we choose $\gamma$ small enough- compared to the worst things that could happen with the 'pollution' $g$. In the no-regret concept, we search for the control $u$, if it exists, which makes things better than $v=0$, for any given perturbation parameter. It is the limit when $\gamma \rightarrow 0$, of the family of low-regret controls $u^{\gamma}$.

This concept is previously introduced by Savage [17] in statistics. Lions was the first to apply it to control distributed systems of incomplete data, motivated by a number of applications in economics, and ecology as well (see for instance [9], [10]).

In [13] (see also [14]), Nakoulima et al. give a precise optimality system (which is a singular optimality system). In [14], the no-regret control for problems of incomplete data, in both the stationary and evolution cases is characterized. A number of applications is given too.

In the litterature mentioned above, the only regular problems are considered. Moreover, the set of controls was a Hilbert space. In this article, we generalize the study to the control of ill-posed problems, where the controls are in a closed convex subset of a Hilbert space only. Without loss of generality, we consider the typical ill-posed problems: the ill-posed backward heat problem.

\subsection{Preliminaries}

Consider an open domain $\Omega \subset \mathbb{R}^{N}$ with smooth boundary $\partial \Omega$, and denote by $Q=(0, T) \times \Omega$, and by $\Sigma=(0, T) \times \partial \Omega$. Then it is well known that the following heat system :

$$
\begin{aligned}
z^{\prime}-\Delta z & \text { in } Q \\
z=0 & \text { on } \Sigma \\
z(0)=0 & \text { in } \Omega
\end{aligned}
$$


is well-posed. Here, $z=z(t, x)$ is the state solution and $v=v(t, x) \in L^{2}(Q)$. Moreover, any solution $z$ of (1)-(3) is a.e. equal to a continuous function from $[0, T]$ to $H^{-1}(\Omega)$ and we have

$$
z^{\prime} \in L^{2}(] 0, T\left[; H^{-2}(\Omega)\right) \quad \text { and } \quad z_{\left.\right|_{\Sigma}} \in H^{-1}(] 0, T\left[; H^{-\frac{1}{2}}(\partial \Omega)\right) .
$$

But, the above system does not admit a solution for arbitrary data (3). Indeed, in the case of final data, i.e. replacing (3) by

$$
z(T)=0 \text { in } \Omega
$$

there is no solution for the backward heat problem, even for regular control $v$, as we can see in the following one-dimensional example.

A counter-example - For $\Omega=] 0, \pi[, T=1$, consider the backward heat system

$$
\left\{\begin{array}{rllll}
\frac{\partial z}{\partial t}-\frac{\partial^{2} z}{\partial x^{2}} & = & v & \text { in } & ] 0, \pi[\times] 0,1[, \\
z(0, t)=z(\pi, t) & = & 0 & \text { in } & ] 0,1[, \\
z(x, 1) & = & 0 & \text { in } & ] 0, \pi[,
\end{array}\right.
$$

where $v \in L^{2}(] 0,1\left[; L^{2}(] 0, \pi[)\right)$ is the uniformly convergent series

$$
v(t, x)=\sqrt{\frac{2}{\pi}} \sum_{m \geq 1} \frac{\sin m x}{m^{2}} .
$$

If $z \in L^{2}(] 0,1\left[; L^{2}(] 0, \pi[)\right)$ is a solution to $(5)$, then $z(t, x)=\sum_{m \geq 1} z_{m}(t) w_{m}(x)$, where $w_{m}(x)=\sqrt{\frac{2}{\pi}} \sin m x$ $\left(w_{m}\right.$ is an eigenfunction for $-\frac{\partial^{2}}{\partial x^{2}}$ related to the eigenvalue $m^{2}$ ), then we have

$$
\left\{\begin{aligned}
\frac{d z_{m}}{d t}(t)+m^{2} z_{m}(t) & \left.=\frac{1}{m^{2}} \quad \text { in } \quad\right] 0,1[ \\
z_{m}(1) & =0
\end{aligned}\right.
$$

so that

$$
z_{m}(t)=\frac{1}{m^{2}} \int_{1}^{t} e^{m^{2}(s-t)} d s=\frac{1}{m^{4}}\left(1-e^{m^{2}(1-t)}\right) .
$$

For every $t \in\left[0,1\left[\right.\right.$, we then obtain $\|z\|_{L^{2}(] 0, \pi[)}^{2}=\sum_{m \geq 1}\left|\frac{1}{m^{4}}\left(1-e^{m^{2}(1-t)}\right)\right|^{2}$. But,

$$
\lim _{m \rightarrow+\infty}\left|\frac{1}{m^{4}}\left(1-e^{m^{2}(1-t)}\right)\right|^{2}=+\infty, \quad \forall t \in[0,1[.
$$

Hence, the series diverges and the solution $z$ of (5) does not exist.

\subsection{Existence of a solution for the ill-posed heat problem}

We consider the following backward heat problem :

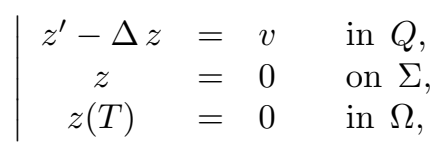

which is a prototype of ill-posed problems. 
Remark 1.1. The backward problem (6) has a unique solution $z$ but for $v$ in a some dense subset of $L^{2}(Q)$. Indeed, for example, we can consider the vector space

$$
V=\left\{w=\sum_{i=1}^{N} \lambda_{i} w_{i}:-\Delta w_{i}=0, w_{i}=0 \text { on } \partial \Omega, \quad \text { and } \quad w_{i} \in L^{2}(\Omega)\right\}
$$

there is $f \in L^{2}(] 0, T[)$ and $w \in V$ such that

$$
v(t, x)=f(t)\left(\sum_{i=1}^{N} \lambda_{i} w_{i}(x)\right)
$$

for given $v \in L^{2}(] 0, T[) \otimes V$ (which is dense in $L^{2}(Q)$ ). It suffices to take $z$ of the form $z(t, x)=\zeta(t) w(x)$, $\zeta=\left(\zeta_{1}, \ldots, \zeta_{N}\right)$. So, $\zeta_{i}$ is solution of

$$
\left\{\begin{aligned}
\frac{\partial \zeta_{i}}{\partial t}-\lambda \zeta_{i} & = & & \text { in }] 0, T[ \\
\zeta_{i}(0) & = & 0 & \text { in } \Omega
\end{aligned}\right.
$$

which defines $\zeta$ in a unique manner.

\subsection{The optimal control question}

Consider $v \in \mathcal{U}_{\mathrm{ad}}, \mathcal{U}_{\mathrm{ad}}$ a non-empty closed convex subset of the Hilbert space of controls $L^{2}(Q)$, and the quadratic function

$$
J(v, z)=\left\|z-z_{d}\right\|_{L^{2}(Q)}^{2}+N\|v\|_{L^{2}(Q)}^{2},
$$

where $z_{d} \in L^{2}(Q), N>0$, and where $\|\cdot\|_{X}$ is the norm on the corresponding Hilbert space $X$.

If a pair $(v, z) \in \mathcal{U}_{a d} \times L^{2}(Q)$ satisfying (6) exists, then it is called a control-state feasible pair. Denote by $\mathcal{X}_{a d}$ the set of admissible pairs for which (6) holds. We suppose in what follows that $\mathcal{X}_{a d}$ is non-empty. Then for every $(v, z) \in \mathcal{X}_{a d}$, we associate the cost function defined by (8), and we consider the optimal control problem :

$$
\inf J(v, z), \quad(v, z) \in \mathcal{U}_{a d} \times L^{2}(\Omega)
$$

which has a unique solution $(u, y)$ that we should characterize.

Lemma 1.2. The problem (9) has one only solution $(u, y)$ called the optimal pair.

Proof. The functional $J: L^{2}(Q) \times L^{2}(Q) \longrightarrow \mathbb{R}$ is a lower semi-continuous function, strictly convex, and coercitive. Hence there is a unique admissible pair $(u, y)$ solution to (9). A classical method to control the system (6) and (8) is the well-known penalization method, which consists in approximating $(u, y)$ by the solution of some penalized problem. More precisely, for $\varepsilon>0$ we define the penalized cost function

$$
J_{\varepsilon}(v, z)=J(v, z)+\frac{1}{2 \varepsilon}\left\|z^{\prime}-\Delta z-v\right\|_{L^{2}(Q)}^{2} .
$$

The optimal pair $\left(u_{\varepsilon}, y_{\varepsilon}\right)$ then converges to $(u, y)$ when $\varepsilon \rightarrow 0$.

The optimality conditions of Euler-Lagrange for $\left(u_{\varepsilon}, y_{\varepsilon}\right)$ are the following :

$$
\frac{d}{d t} J_{\varepsilon}\left(u_{\varepsilon}, y_{\varepsilon}+t\left(z-y_{\varepsilon}\right)\right)_{\left.\right|_{t=0}}=0, \quad \forall z \in \mathcal{F}
$$


and

$$
\frac{d}{d t} J_{\varepsilon}\left(u_{\varepsilon}+t\left(v-u_{\varepsilon}\right), y_{\varepsilon}\right)_{\mid t=0} \geq 0 \quad \forall v \in \mathcal{U}_{a d},
$$

then an optimality system is obtained by the introduction of the adjoint state

$$
p_{\varepsilon}=-\frac{1}{\varepsilon}\left(y_{\varepsilon}^{\prime}-\Delta y_{\varepsilon}-u_{\varepsilon}\right)
$$

A priori estimates (consisting in bounding $p_{\varepsilon}$ in $L^{2}(Q)$ ) have to be obtained, which allows the passage to the limit under some hypothesis : For the problem (6) and (8)-(9), J. L. Lions obtained in [6] a singular optimality system, under the extra hypothesis of Slater type :

$$
\mathcal{U}_{\text {ad }} \text { has a non-empty interior. }
$$

More precisely, we have the following theorem due to Lions :

Theorem 1.3. (J.L. Lions [6]) Under hypothesis (12), there is a unique $(u, y, p) \in \mathcal{U}_{a d} \times L^{2}(Q) \times L^{2}(Q)$, solution to the optimal control problem (6) and (8)-(9). Moreover, this solution is characterized by the following singular optimality system (SOS) :

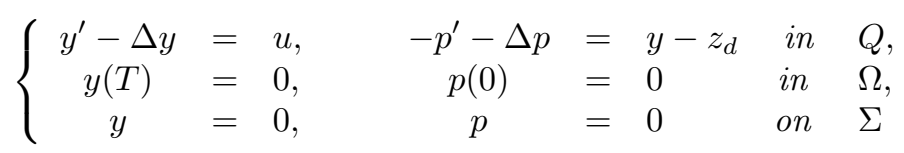

with the variational inequality

$$
(p+N u, v-u)_{L^{2}(Q)} \geq 0 \quad \forall v \in \mathcal{U}_{a d} .
$$

Remark 1.4. Of course when $\mathcal{U}_{a d}=L^{2}(Q)$, the hypothesis (12) is satisfied and then the above theorem holds. In some applications, the Slater hypothesis (12) is not satisfied, as when $\mathcal{U}_{a d}=\left(L^{2}(Q)\right)^{+}$which has an empty interior. In the following, we propose alternative approach which does not assume (12).

\section{The REgUlarization APPROACH}

In this section, some elliptic regularization of the ill-posed parabolic problem (6) is proposed. We obtain some well-posed problem but with a new unknown datum. We then let the classical control notion away, to consider the one of no-regret control by Lions [8], and recently developed by Nakoulima et al. in [13] [14].

For any $\varepsilon>0$, we consider the regularized problem :

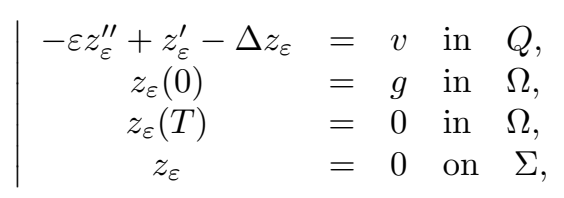

where $g \in L^{2}(\Omega)$.

It is clear that for any $\varepsilon>0$, and any data $(v, g)$, there is a unique state solution $z_{\varepsilon}=z_{\varepsilon}(v, g)$ of the elliptic problem (15) (see Lions-Magenes [12] for example), for which we associate the cost given by :

$$
J_{\mathcal{\varepsilon}}(v, g)=\left\|z_{\mathcal{E}}(v, g)-z_{d}\right\|_{L^{2}(Q)}^{2}+N\|v\|_{L^{2}(Q)}^{2}, \quad g \in L^{2}(\Omega) .
$$


We are concerned with the optimal control of the problem (15)-(16). Clearly we want

$$
\inf _{v \in \mathcal{U}_{a d}} J_{\varepsilon}(v, g) \quad \forall g \in L^{2}(\Omega) .
$$

If $g \in G \subset L^{2}(\Omega)$ with $G$ finite, then we can give a Pareto sense to (17). But if $G$ is not finite, then the above minimization problem has no sense. One natural idea is to consider the following minimization problem :

$$
\inf _{v \in \mathcal{U}_{a d}}\left(\sup _{g \in L^{2}(\Omega)} J_{\varepsilon}(v, g)\right)
$$

but from above, we see that $J_{\varepsilon}$ is not bounded, $\operatorname{since} \sup _{g \in L^{2}(\Omega)} J_{\varepsilon}(v, g)=+\infty$. The idea of Lions is then to look for controls $v$-if they exist- such that

$$
J_{\varepsilon}(v, g) \leq J_{\mathcal{\varepsilon}}(0, g) \quad \forall g \in L^{2}(\Omega),
$$

and thus

$$
J_{\varepsilon}(v, g)-J_{\varepsilon}(0, g) \leq 0 \quad \forall g \in L^{2}(\Omega) .
$$

Those controls doing better than $v=0$ for every pollution $g$ are called no-regret controls.

Definition 2.1. We say that $u \in \mathcal{U}_{\text {ad }}$ is a no-regret control for (15)-(16) if $u$ is a solution to the following problem:

$$
\inf _{v \in \mathcal{U}_{a d}}\left(\sup _{g \in L^{2}(\Omega)}\left(J_{\varepsilon}(v, g)-J_{\varepsilon}(0, g)\right)\right) .
$$

Lemma 2.2. For any $v \in \mathcal{U}_{a d}$ we have

$$
J_{\varepsilon}(v, g)-J_{\varepsilon}(0, g)=J_{\varepsilon}(v, 0)-J_{\varepsilon}(0,0)+2\left\langle\xi_{\varepsilon}^{\prime}(0), g\right\rangle_{L^{2}(\Omega)} \quad \forall g \in L^{2}(\Omega),
$$

where $\xi_{\varepsilon}$ satisfies to :

$$
-\xi_{\varepsilon}^{\prime}-\varepsilon \xi_{\varepsilon}^{\prime \prime}-\Delta \xi_{\varepsilon}=y_{\varepsilon}(v, 0) \text { in } Q, \quad \xi_{\varepsilon}(0)=\xi_{\varepsilon}(T)=0 \text { in } \Omega, \quad \xi_{\varepsilon}=0 \text { on } \Sigma .
$$

Proof. A direct computation gives

$$
J_{\varepsilon}(v, g)-J_{\varepsilon}(0, g)=J_{\varepsilon}(v, 0)-J_{\varepsilon}(0,0)+2\left\langle z_{\varepsilon}(v, 0) ; z_{\varepsilon}(0, g)\right\rangle_{L^{2}(Q)} .
$$

Following the the Green formula we get

$$
\left\langle z_{\varepsilon}(v, 0) ; z_{\varepsilon}(0, g)\right\rangle=\varepsilon\left\langle\xi_{\varepsilon}{ }^{\prime}(0) ; g\right\rangle_{L^{2}(Q)},
$$

where $\xi_{\varepsilon}$ is given by $(20)$.

Remark 2.3. Of course the problem (18) is defined only for the controls $v \in \mathcal{U}_{\text {ad }}$ such that

$$
\sup _{g \in L^{2}(\Omega)}\left(J_{\varepsilon}(v, g)-J_{\varepsilon}(0, g)\right)<\infty .
$$

From (19) this is achieved iff $v \in K$, where $K=\left\{w \in \mathcal{U}_{\mathrm{ad}},\left\langle\xi_{\varepsilon}(w), g\right\rangle=0 \forall g \in L^{2}(\Omega)\right\}$. This set is hard to characterize. As in [13], for any $\gamma>0$, we define the control in the low-regret sense. 
Definition 2.4. The low-regret control for (15)-(16), is the solution to the following perturbed system :

$$
\inf _{v \in \mathcal{U}_{\mathrm{ad}}}\left(\sup _{g \in L^{2}(\Omega)}\left(J_{\varepsilon}(v, g)-J_{\varepsilon}(0, g)-\gamma\|g\|_{L^{2}(\Omega)}^{2}\right)\right) .
$$

Remark that :

$$
\begin{aligned}
& \inf _{v \in \mathcal{U}_{\mathrm{ad}}}\left(\sup _{g \in L^{2}(\Omega)}\left(J_{\varepsilon}(v, g)-J_{\varepsilon}(0, g)-\gamma\|g\|_{L^{2}(\Omega)}^{2}\right)\right) \\
& \quad=\inf _{v \in \mathcal{U}_{\mathrm{ad}}}\left(J_{\varepsilon}(v, 0)-J_{\varepsilon}(0,0)+\left(\sup _{g \in L^{2}(\Omega)} 2\left\langle z_{\varepsilon}(v, 0) ; z_{\varepsilon}(0, g)\right\rangle_{L^{2}(Q)}-\gamma\|g\|_{L^{2}(\Omega)}^{2}\right)\right) .
\end{aligned}
$$

Thanks to Legendre transform, we get the classical control problem :

$$
\inf _{v \in \mathcal{U}_{\mathrm{ad}}} \mathcal{J}_{\varepsilon}^{\gamma}(v)
$$

where

$$
\mathcal{J}_{\varepsilon}^{\gamma}(v)=J_{\varepsilon}(v, 0)-J_{\varepsilon}(0,0)+\frac{\varepsilon^{2}}{\gamma}\left\|\xi_{\varepsilon}^{\prime}(T, v)\right\|_{L^{2}(\Omega)}^{2}
$$

and where $\xi_{\varepsilon}$ satisfies $(20)$.

Remark 2.5. As we can see here, the low-regret control method allows to transform systematically problems with uncertainty into standard control problems. We can then use the Euler-Lagrange method.

We can replace now (21) by (22) and (23) for the low-regret control.

Lemma 2.6. The problem (15) with control (22)-(23) has one only solution $u_{\varepsilon}^{\gamma}$, called the 'approximate' lowregret control.

Proof. We have $\mathcal{J}_{\varepsilon}^{\gamma}(v) \geq-J_{\varepsilon}(0,0)=-\left\|z_{d}\right\|_{L^{2}(\Omega)}^{2} \forall v \in \mathcal{U}_{\text {ad }}$. Then $d=\inf _{v \in \mathcal{U}_{\text {ad }}} \mathcal{J}_{\varepsilon}^{\gamma}(v)>-\infty$. Let $v_{n}$ be some minimizing sequence (such that $d=\lim _{n \rightarrow \infty} \mathcal{J}_{\varepsilon}^{\gamma}\left(v_{n}\right)$ ). We have

$$
-\left\|z_{d}\right\|_{L^{2}(\Omega)}^{2} \leq \mathcal{J}_{\varepsilon}^{\gamma}\left(v_{n}\right)=J_{\varepsilon}\left(v_{n}, 0\right)-J_{\varepsilon}(0,0)+\frac{1}{\gamma}\left\|\xi_{\varepsilon}{ }^{\prime}(0)\right\|_{L^{2}(\Omega)}^{2} \leq d_{\gamma}+1
$$

Then we deduce the bounds

$$
\left\|v_{n}\right\|_{L^{2}(Q)} \leq c, \quad \frac{1}{\sqrt{\gamma}}\left\|\xi_{\varepsilon}^{\prime}\left(v_{n}\right)(0)\right\|_{L^{2}(\Omega)} \leq c, \quad\left\|y_{\varepsilon}\left(v_{n}, 0\right)-z_{d}\right\|_{L^{2}(Q)} \leq c,
$$

where the constant $c$ is independent of $n$.

There exists $u_{\varepsilon}^{\gamma} \in \mathcal{U}_{\text {ad }}$ such that $v_{n} \rightarrow u_{\varepsilon}^{\gamma}$ weakly in $\mathcal{U}_{\text {ad }}$ (which is closed). Also, $y_{\varepsilon}\left(v_{n}, 0\right) \rightarrow y_{\varepsilon}\left(u_{\varepsilon}^{\gamma}, 0\right)$ (continuity w.r.t the data). Now since $\mathcal{J}_{\varepsilon}^{\gamma}$ is strictly convex, $u_{\varepsilon}^{\gamma}$ is unique. 
Proposition 2.7. The 'approximate' low-regret control $u_{\varepsilon}^{\gamma}$ is characterized by the unique quadruplet $\left\{u_{\varepsilon}^{\gamma}, y_{\varepsilon}^{\gamma}, \rho_{\varepsilon}^{\gamma}, p_{\varepsilon}^{\gamma}\right\}$, solution to the system :

$$
\mid \begin{aligned}
& y_{\varepsilon}^{\gamma^{\prime}}-\varepsilon y_{\varepsilon}^{\gamma \prime \prime}-\Delta y_{\varepsilon}^{\gamma}=u_{\varepsilon}^{\gamma}, \quad \rho_{\varepsilon}^{\gamma^{\prime}}-\varepsilon \rho_{\varepsilon}^{\gamma^{\prime \prime}}-\Delta \rho_{\varepsilon}^{\gamma}=0, \quad \text { and } \\
& -p_{\varepsilon}^{\gamma \prime}-\varepsilon p_{\varepsilon}^{\gamma \prime \prime}-\Delta p_{\varepsilon}^{\gamma}=y_{\varepsilon}^{\gamma}-z_{d}+\rho_{\varepsilon}^{\gamma} \quad \text { in } Q, \\
& y_{\varepsilon}^{\gamma}(0)=y_{\varepsilon}^{\gamma}(T)=0, \quad \rho_{\varepsilon}^{\gamma}(T)=0, \quad \rho_{\varepsilon}^{\gamma}(0)=\frac{\varepsilon}{\gamma} \xi_{\varepsilon}^{\gamma^{\prime}}(0), \\
& p_{\varepsilon}^{\gamma}(0)=p_{\varepsilon}^{\gamma}(T)=0 \quad \text { in } \Omega, \\
& y_{\varepsilon}^{\gamma}=0, \quad \rho_{\varepsilon}^{\gamma}=0, \quad p_{\varepsilon}^{\gamma}=0 \quad \text { on } \Sigma,
\end{aligned}
$$

with (20),

and the variational inequality :

$$
\left\langle p_{\varepsilon}^{\gamma}+N u_{\varepsilon}^{\gamma}, v-u_{\varepsilon}^{\gamma}\right\rangle \geq 0, \quad \forall v \in \mathcal{U}_{a d}
$$

Proof. The first order Euler condition for (22) and (23) gives :

$$
\left\langle y_{\varepsilon}^{\gamma}-z_{d}, y_{\varepsilon}(w, 0)\right\rangle_{L^{2}(Q) \times L^{2}(Q)}+N\left\langle u_{\varepsilon}^{\gamma}, w\right\rangle_{L^{2}(Q) \times L^{2}(Q)}+\left\langle\frac{\varepsilon^{2}}{\gamma} \xi_{\varepsilon}^{\gamma^{\prime}}(0), \xi_{\varepsilon}^{\prime}(0, w)\right\rangle_{L^{2}(\Omega) \times L^{2}(\Omega)} \geq 0
$$

where $y_{\varepsilon}^{\gamma}=y_{\varepsilon}\left(u_{\varepsilon}^{\gamma}, 0\right)$, and $\xi_{\varepsilon}^{\gamma}=\xi_{\varepsilon}\left(u_{\varepsilon}^{\gamma}, 0\right)$. We then define $\rho_{\varepsilon}^{\gamma}=\rho_{\varepsilon}\left(u_{\varepsilon}^{\gamma}, 0\right)$ which is the solution of $\rho_{\varepsilon}^{\gamma^{\prime}}-\varepsilon \rho_{\varepsilon}^{\gamma^{\prime \prime}}-\Delta \rho_{\varepsilon}^{\gamma}=0, \rho_{\varepsilon}^{\gamma}(0)=(\varepsilon / \gamma) \xi_{\varepsilon}^{\gamma^{\prime}}(T), \rho_{\varepsilon}^{\gamma}(T)=0$, and $\rho_{\varepsilon}^{\gamma}=0$ on $\Sigma$, such that :

$$
\left\langle\frac{\varepsilon^{2}}{\gamma} \xi_{\varepsilon}^{\gamma^{\prime}}(0), \xi_{\varepsilon}^{\prime}(0, w)\right\rangle_{L^{2}(\Omega) \times L^{2}(\Omega)}=\left\langle\varepsilon \rho_{\varepsilon}^{\gamma}(0), \xi_{\varepsilon}^{\prime}(0, w)\right\rangle_{L^{2}(\Omega) \times L^{2}(\Omega)}=\left\langle\rho_{\varepsilon}^{\gamma}, y_{\varepsilon}(w, 0)\right\rangle_{L^{2}(Q) \times L^{2}(Q)}
$$

by the Green formula.

Introduce now the adjoint state $p_{\varepsilon}^{\gamma}=p_{\varepsilon}\left(u_{\varepsilon}^{\gamma}, 0\right)$ as follows : we solve $-p_{\varepsilon}^{\gamma \prime}-\varepsilon p_{\varepsilon}^{\gamma \prime \prime}-\Delta p_{\varepsilon}^{\gamma}=y_{\varepsilon}^{\gamma}-z_{d}+\rho_{\varepsilon}^{\gamma}$, $p_{\varepsilon}^{\gamma}(T)=p_{\varepsilon}^{\gamma}(0)=0$, and $p_{\varepsilon}^{\gamma}=0$ on $\Sigma$. Hence we have

$$
\left\langle y_{\varepsilon}^{\gamma}-z_{d}+\rho_{\varepsilon}^{\gamma}, y_{\varepsilon}(w, 0)\right\rangle_{L^{2}(Q) \times L^{2}(Q)}=\left\langle p_{\varepsilon}^{\gamma}, w\right\rangle_{L^{2}(Q) \times L^{2}(Q)}
$$

Finally,

$$
\left\langle p_{\varepsilon}^{\gamma}+N u_{\varepsilon}^{\gamma}, w\right\rangle_{L^{2}(Q) \times L^{2}(Q)} \geq 0
$$

\subsection{A priori estimates. The low-regret control}

In this section we give the S.O.S for the low-regret control of the backward heat equation. Estimates are given in the following statement :

Proposition 2.8. There is some positive constant $C$, and, for any small $\eta>0$, there is some constant $C_{\eta}>0$ such that :

$$
\begin{aligned}
& \left\|u_{\varepsilon}^{\gamma}\right\|_{L^{2}(Q)} \leq C, \quad\left\|y_{\varepsilon}^{\gamma}\right\|_{L^{2}(Q)} \leq C, \quad \frac{\varepsilon}{\sqrt{\gamma}}\left\|\xi_{\varepsilon}^{\gamma^{\prime}}(0)\right\|_{L^{2}(\Omega)} \leq C, \\
& \varepsilon\left\|y_{\varepsilon}^{\gamma^{\prime}}\right\|_{L^{2}(Q)}+\left\|y_{\varepsilon}^{\gamma}\right\|_{L^{2}(Q)} \leq C, \quad\left\|y_{\varepsilon}^{\gamma^{\prime}}\right\|_{L^{2}(] 0, T-\eta\left[; H^{-1}(\Omega)\right)} \leq C_{\eta},
\end{aligned}
$$


and,

$$
\begin{aligned}
\varepsilon\left\|\xi_{\varepsilon}^{\gamma^{\prime}}\right\|_{L^{2}(Q)}+\left\|\xi_{\varepsilon}^{\gamma}\right\|_{L^{2}(Q)} \leq C, & \left\|\xi_{\varepsilon}^{\gamma^{\prime}}\right\|_{L^{2}(] \eta, T\left[; H^{-1}(\Omega)\right)} & \leq C_{\eta}, \\
\varepsilon\left\|\rho_{\varepsilon}^{\gamma^{\prime}}\right\|_{L^{2}(Q)}+\left\|\rho_{\varepsilon}^{\gamma}\right\|_{L^{2}(Q)} \leq C, & \left\|\rho_{\varepsilon}^{\gamma^{\prime}}\right\|_{L^{2}(] 0, T-\eta\left[; H^{-1}(\Omega)\right)} & \leq C_{\eta}, \\
\varepsilon\left\|p_{\varepsilon}^{\gamma^{\prime}}\right\|_{L^{2}(Q)}+\left\|p_{\varepsilon}^{\gamma}\right\|_{L^{2}(Q)} \leq C, & \left\|p_{\varepsilon}^{\gamma^{\prime}}\right\|_{L^{2}(] \eta, T\left[; H^{-1}(\Omega)\right)} & \leq C_{\eta} .
\end{aligned}
$$

Proof. Since $u_{\varepsilon}^{\gamma}$ is the approximate low-regret control

$$
\mathcal{J}_{\varepsilon}^{\gamma}\left(u_{\varepsilon}^{\gamma}\right) \leq \mathcal{J}_{\varepsilon}^{\gamma}(v) \quad \forall v \in \mathcal{U}_{a d},
$$

in the particular case where $v=0$,

$$
J_{\varepsilon}\left(u_{\varepsilon}^{\gamma}, 0\right)-J_{\varepsilon}(0,0)+\frac{\varepsilon^{2}}{\gamma}\left\|\xi_{\varepsilon}{ }^{\prime}\left(u_{\varepsilon}^{\gamma}\right)(0)\right\|_{L^{2}(\Omega)}^{2} \leq \frac{\varepsilon^{2}}{\gamma}\left\|\xi_{\varepsilon}{ }^{\prime}\left(u_{\varepsilon}^{\gamma}\right)(0)\right\|_{L^{2}(\Omega)}^{2} .
$$

On the other hand $y_{\varepsilon}(0,0)(t, x)=\xi_{\varepsilon}(0)(t, x)=0$ in $[0, T] \times \bar{\Omega}$, then :

$$
\left\|y_{\varepsilon}\left(u_{\varepsilon}^{\gamma}, 0\right)-z_{d}\right\|_{L^{2}(Q)}^{2}+N\left\|u_{\varepsilon}^{\gamma}\right\|_{L^{2}(Q)}^{2}+\left\|\frac{\varepsilon}{\sqrt{\gamma}} \xi_{\varepsilon}^{\prime}\left(u_{\varepsilon}^{\gamma}\right)(0)\right\|_{L^{2}(\Omega)}^{2} \leq\left\|z_{d}\right\|_{L^{2}(Q)}^{2}=\text { constant }
$$

so (24) holds.

From the Poincaré formula, there is some constant $C_{1}>0$ such that:

$$
\left(\varepsilon\left\|\frac{\partial y_{\varepsilon}^{\gamma}}{\partial t}\right\|_{L^{2}(Q)}+\left\|y_{\varepsilon}^{\gamma}\right\|_{L^{2}(Q)}\right)\left\|y_{\varepsilon}^{\gamma}\right\|_{L^{2}(Q)} \leq C_{1}\left\|u_{\varepsilon}^{\gamma}\right\|_{L^{2}(Q)}\left\|y_{\varepsilon}^{\gamma}\right\|_{L^{2}(Q)},
$$

thus, there is some constant $C_{2}>0$ such that

$$
\varepsilon\left\|\frac{\partial y_{\varepsilon}^{\gamma}}{\partial t}\right\|_{L^{2}(Q)}+\left\|y_{\varepsilon}^{\gamma}\right\|_{L^{2}(Q)} \leq C_{2}
$$

that is the first part of (25). Now, we consider the equation $-\varepsilon \frac{\partial^{2} y_{\varepsilon}}{\partial t^{2}}+\frac{\partial y_{\varepsilon}}{\partial t}=v+\Delta y_{\varepsilon}$, and denote by $g_{\varepsilon}=v+\Delta y_{\varepsilon}$. Then $g_{\varepsilon}$ stays in some bounded subset of $L^{2}(Q)$.

Consider some function $\varphi \in C^{1}([0, T])$ such that $\varphi(0)=1$ and $\varphi(T)=0$. Multiply both members of

$$
-\varepsilon \frac{\partial^{2} y_{\varepsilon}}{\partial t^{2}}+\frac{\partial y_{\varepsilon}}{\partial t}=g_{\varepsilon}
$$

by $\varphi \frac{\partial y_{\varepsilon}}{\partial t}$ and integrate over the whole set $Q$. We have

$$
-\frac{\varepsilon}{2} \int_{0}^{T} \varphi \frac{d}{d t}\left\|\frac{\partial y_{\varepsilon}}{\partial t}\right\|_{L^{2}(\Omega)}^{2} d t+\int_{0}^{T} \varphi\left\|\frac{\partial y_{\varepsilon}}{\partial t}\right\|_{L^{2}(\Omega)}^{2} d t=\int_{0}^{T} \varphi\left(g_{\varepsilon}, \frac{\partial y_{\varepsilon}}{\partial t}\right)_{L^{2}(\Omega)} d t
$$

And,

$$
\int_{0}^{T} \varphi \frac{d}{d t}\left\|\frac{\partial y_{\varepsilon}}{\partial t}\right\|_{L^{2}(\Omega)}^{2} d t=-\left\|\frac{\partial y_{\varepsilon}}{\partial t}(0)\right\|_{L^{2}(\Omega)}^{2}-\int_{0}^{T} \frac{d \varphi}{d t}\left\|\frac{\partial y_{\varepsilon}}{\partial t}\right\|_{L^{2}(\Omega)}^{2} d t
$$


thus

$$
\frac{\varepsilon}{2}\left\|\frac{\partial y_{\varepsilon}}{\partial t}(0)\right\|_{L^{2}(\Omega)}^{2}+\frac{\varepsilon}{2} \int_{0}^{T} \frac{d \varphi}{d t}\left\|\frac{\partial y_{\varepsilon}}{\partial t}\right\|_{L^{2}(\Omega)}^{2} d t+\int_{0}^{T} \varphi\left\|\frac{\partial y_{\varepsilon}}{\partial t}\right\|_{L^{2}(\Omega)}^{2} d t=\int_{0}^{T} \varphi\left(g_{\varepsilon}, \frac{\partial y_{\varepsilon}}{\partial t}\right)_{L^{2}(\Omega)} d t .
$$

The second term is $O(1)$, so

$$
\int_{0}^{T} \varphi\left\|\frac{\partial y_{\varepsilon}}{\partial t}\right\|_{L^{2}(\Omega)}^{2} d t=\int_{0}^{T} \varphi\left(g_{\varepsilon}, \frac{\partial y_{\varepsilon}}{\partial t}\right)_{L^{2}(\Omega)} d t+O(1)
$$

We finally deduce (25) from the triangular inequality.

The estimates (26), (27) and (28) follow easily.

Theorem 2.9. The low-regret control $u^{\gamma}=\lim _{\varepsilon \rightarrow 0} u_{\varepsilon}^{\gamma}$ for the backwards heat equation (6) is characterized by the unique $\left\{u^{\gamma}, y^{\gamma}, \xi^{\gamma}, \rho^{\gamma}, p^{\gamma}\right\}$, solution to the system :

$$
\mid \begin{aligned}
& y^{\gamma^{\prime}}-\Delta y^{\gamma}=u^{\gamma}, \quad-\xi^{\gamma^{\prime}}-\Delta \xi^{\gamma}=y^{\gamma}, \quad \rho^{\gamma^{\prime}}-\Delta \rho^{\gamma}=0, \quad \text { and } \\
& -p^{\gamma^{\prime}}-\Delta p^{\gamma}=y^{\gamma}-z_{d}+\rho^{\gamma} \text { in } Q, \\
& y^{\gamma}(0)=0, \quad \xi^{\gamma}(T)=0, \quad \text { and } \\
& \rho^{\gamma}(0)=\lambda^{\gamma}(0), \quad p^{\gamma}(T)=0 \quad \text { in } \Omega, \\
& y^{\gamma}=0, \quad \xi^{\gamma}=0, \quad \rho^{\gamma}=0, \quad p^{\gamma}=0 \quad \text { on } \Sigma
\end{aligned}
$$

with the following weak limits

and the variational inequality :

$$
y^{\gamma}=\lim _{\varepsilon \rightarrow 0} y_{\varepsilon}^{\gamma} \quad, \xi^{\gamma}=\lim _{\varepsilon \rightarrow 0} \xi_{\varepsilon}^{\gamma} \quad, \rho^{\gamma}=\lim _{\varepsilon \rightarrow 0} \rho_{\varepsilon}^{\gamma} \quad, p^{\gamma}=\lim _{\varepsilon \rightarrow 0} p_{\varepsilon}^{\gamma},
$$

where

$$
\left\langle p^{\gamma}+N u^{\gamma}, v-u^{\gamma}\right\rangle \geq 0 \quad \forall v \in \mathcal{U}_{a d}
$$

$$
u^{\gamma}, y^{\gamma}, p^{\gamma}, \rho^{\gamma}, \xi^{\gamma} \in L^{2}(] 0, T\left[; L^{2}(\Omega)\right), \quad \lambda^{\gamma}(0) \in L^{2}(\Omega)
$$

Proof. We use the estimates of proposition 2.8. ¿From (29), we deduce the following limits :

$$
\begin{array}{cll}
u_{\varepsilon}^{\gamma} & \rightarrow u^{\gamma} & \text { weakly in } \mathcal{U}_{a d}, \\
y_{\varepsilon}^{\gamma} & \rightarrow y^{\gamma} & \text { weakly in } L^{2}(Q), \\
\frac{\varepsilon}{\sqrt{\gamma}} \frac{\partial \xi_{\varepsilon}\left(u_{\varepsilon}^{\gamma}\right)}{\partial t}(0) & \rightarrow \lambda^{\gamma}(0) & \text { weakly in } L^{2}(\Omega),
\end{array}
$$

(up to extract a subsequences $\left(u_{\varepsilon}^{\gamma}\right),\left(y_{\varepsilon}^{\gamma}\right)$ and $\left.\left(\frac{\varepsilon}{\sqrt{\gamma}} \frac{\partial \xi_{\varepsilon}\left(u_{\varepsilon}^{\gamma}\right)}{\partial t}(T)\right)\right)$.

For fixed $\gamma>0$, the adjoint state $p_{\varepsilon}^{\gamma}$ depends boundedly on $\varepsilon$ (from (28)).

\subsection{Zero-order corrector}

For the no-regret optimal control to the original problem, we now introduce the notion of corrector of order 0 of Lions [7] for elliptic regularizations, instead of using the Slater hypothesis (12). Indeed, as it is well known, the passage to the limit gives no information on $y^{\gamma}(T)$. Moreover, $y^{\gamma}(T) \neq 0$ in general (see Dorville [1] for details).

We assume the following :

$$
y^{\gamma}(T) \in H_{0}^{1}(\Omega), y^{\gamma^{\prime}} \in L^{2}(Q) .
$$

Denote by

$$
\mathcal{V}=\left\{\varphi \in L^{2}(] 0, T\left[; H_{0}^{1}(\Omega)\right) \text { such that } \varphi^{\prime} \in L^{2}(Q)\right\}
$$


and by

$$
\mathcal{V}_{0}=\{\varphi \in \mathcal{V} \text { such that } \varphi(0)=0, \varphi(T)=0\} .
$$

Definition 2.10. We say that a function $\theta_{\varepsilon}^{\gamma} \in \mathcal{V}$ is a corrector of order 0 iff

$$
\begin{array}{cc}
\varepsilon\left(\theta_{\varepsilon}^{\gamma^{\prime}}, \varphi^{\prime}\right)_{L^{2}(Q)}+\left(\theta_{\varepsilon}^{\gamma^{\prime}}, \varphi\right)_{L^{2}(Q)}+\left(\nabla \theta_{\varepsilon}^{\gamma}, \nabla \varphi\right)_{L^{2}(Q)} & =\sqrt{\varepsilon}\left(f_{\varepsilon}, \varphi\right)_{L^{2}(Q)} \quad \forall \mathcal{V}_{0}, \\
\theta_{\varepsilon}^{\gamma}(T)+y^{\gamma}(T) & =0,
\end{array}
$$

where we suppose that

$$
\left\|f_{\varepsilon}\right\|_{L^{2}(] 0, T\left[; H^{-1}(\Omega)\right)} \leq C .
$$

Getting a zero-order corrector We recall how to calculate a corrector of order 0 :

We define $\varphi_{\varepsilon}^{\gamma}$ by writing

$$
\left\{\begin{array}{ccc}
-\varepsilon \varphi_{\varepsilon}^{\gamma \prime \prime}+\varphi_{\varepsilon}^{\gamma^{\prime}} & = & 0 \\
\varphi_{\varepsilon}^{\gamma}(T) & = & -y^{\gamma}(T),
\end{array}\right.
$$

$\varphi_{\varepsilon}^{\gamma}$ decreasing rapidly when $t \rightarrow-\infty$,

then

$$
\theta_{\varepsilon}^{\gamma}(t)=-y^{\gamma}(T) e^{-\frac{T-t}{\varepsilon}}
$$

If we suppose that $y^{\gamma}(T) \in H_{0}^{1}(\Omega)$, the function

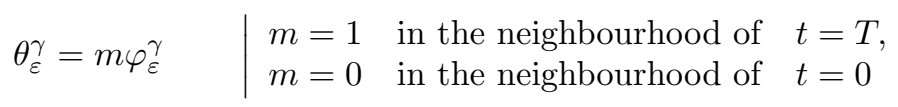

is a corrector of order 0 .

We then satisfy the variational equation, the main term being

$$
m \Delta y^{\gamma}(T) e^{-\frac{T-t}{\varepsilon}}=\sqrt{\varepsilon} h_{\varepsilon}^{\gamma} .
$$

Hence, under the above hypothesis we have :

$$
\int_{0}^{T}\left\|h_{\varepsilon}^{\gamma}\right\|_{H^{-1}(\Omega)}^{2} d t \leq C \varepsilon^{-1} \int_{0}^{T} e^{-\frac{2(T-t)}{\varepsilon}} d t=o(1) .
$$

We then have the theorem :

Theorem 2.11. Let be $\theta_{\varepsilon}^{\gamma}$ a corrector of order 0 defined by (33) and (34). We then have

$$
\left\|y_{\varepsilon}^{\gamma}-\left(y^{\gamma}+\theta_{\varepsilon}^{\gamma}\right)\right\|_{L^{2}(] 0, T\left[; H_{0}^{1}\right)} \leq C \sqrt{\varepsilon} .
$$

Moreover,

when $\varepsilon$ tends to 0 .

$$
\frac{d}{d t}\left[y_{\varepsilon}^{\gamma}-\left(y^{\gamma}+\theta_{\varepsilon}^{\gamma}\right)\right] \rightarrow 0 \quad \text { weakly in } \quad L^{2}(Q),
$$

Proof. If we put $w_{\varepsilon}^{\gamma}=y_{\varepsilon}^{\gamma}-\left(y^{\gamma}+\theta_{\varepsilon}^{\gamma}\right)$, then

$$
\begin{gathered}
\varepsilon\left(w_{\varepsilon}^{\gamma^{\prime}}, \varphi^{\prime}\right)_{L^{2}(Q)}+\left(w_{\varepsilon}^{\gamma^{\prime}}, \varphi\right)_{L^{2}(Q)}+\left(\nabla w_{\varepsilon}^{\gamma}, \nabla \varphi\right)_{L^{2}(Q)} \\
=-\varepsilon\left(y^{\gamma^{\prime}}, \varphi^{\prime}\right)_{L^{2}(Q)}-\sqrt{\varepsilon}\left(f_{\varepsilon}, \varphi\right)_{L^{2}(Q)} \quad \forall \mathcal{V}_{0} .
\end{gathered}
$$


Particularly, if $\varphi=w_{\varepsilon}^{\gamma}$, then

$$
\varepsilon\left\|w_{\varepsilon}^{\gamma^{\prime}}\right\|_{L^{2}(Q)}^{2}+\left\|w_{\varepsilon}^{\gamma}\right\|_{L^{2}(Q)}^{2} \leq C \sqrt{\varepsilon}\left[\sqrt{\varepsilon}\left\|w_{\varepsilon}^{\gamma^{\prime}}\right\|_{L^{2}(Q)}+\left\|w_{\varepsilon}^{\gamma}\right\|_{L^{2}(Q)}\right] .
$$

Thus the inequalities (36) and (37) hold.

\subsection{Passage to the limit}

We use the regularity properties of the heat equation, the - well posed- one, as follows :

First, we notice that $\left\|y_{\gamma}\right\|_{L^{2}(Q)} \leq C$ by the above proposition. It remains to see that $\xi_{\gamma}\left(\right.$ resp. $\left.p_{\gamma}\right)$ formally satisfies to the well-posed system :

$$
\left.(*) \mid \begin{array}{l|l}
-\xi^{\gamma^{\prime}}-\Delta \xi^{\gamma}=y^{\gamma} \in L^{2}(Q), \\
\xi^{\gamma}=0, \\
\xi^{\gamma}(T)=0,
\end{array} \quad \text { resp. (**) } \mid \begin{array}{l}
-p^{\gamma^{\prime}}-\Delta p^{\gamma}=y^{\gamma}-z_{d}, \\
p^{\gamma}=0 \text { on } \Sigma, \\
p^{\gamma}(T)=0,
\end{array}\right)
$$

with the mean of a zero corrector. But $(*)$ implies that

$$
\left\|\xi^{\gamma}\right\|_{L^{2}\left(0, T ; H_{0}^{1}(\Omega)\right)}+\left\|\xi^{\gamma^{\prime}}\right\|_{L^{2}\left(0, T ; H^{-1}(\Omega)\right)} \leq C,
$$

(resp. (**) gives $\left\|p^{\gamma}\right\|_{L^{2}\left(0, T ; H_{0}^{1}(\Omega)\right)}+\left\|p^{\gamma^{\prime}}\right\|_{L^{2}\left(0, T ; H^{-1}(\Omega)\right)} \leq C$.)

Then $\xi^{\gamma} \rightarrow \xi$ (resp. $p^{\gamma} \rightarrow p$ ) weakly in $L^{2}\left(0, T ; H_{0}^{1}(\Omega)\right.$ ), and by compactness $\xi^{\gamma} \rightarrow \xi$ (resp. $p^{\gamma} \rightarrow p$ ) stronly in $L^{2}\left(0, T ; L^{2}(\Omega)\right)$.

Also,

$$
\mid \begin{aligned}
& \rho^{\gamma^{\prime}}-\Delta \rho^{\gamma}=0 \\
& \rho^{\gamma}=0 \\
& \rho^{\gamma}(0)=\lambda^{\gamma}(0)
\end{aligned}
$$

implies that $\rho^{\gamma} \rightarrow \rho$ strong in $L^{2}\left(0, T ; L^{2}(\Omega)\right)$ by the same arguments, because

$$
\left\|\lambda^{\gamma}(0)\right\|_{L^{2}(\Omega)} \leq \lim _{\varepsilon \rightarrow 0} \frac{\varepsilon}{\sqrt{\gamma}}\left\|\xi_{\varepsilon}^{\prime}\left(u_{\varepsilon}^{\gamma}\right)(0)\right\|_{L^{2}(\Omega)} \leq C, \quad \text { then } \quad \lambda^{\gamma}(0) \rightarrow \lambda(0) \in L^{2}(\Omega)
$$

We then can announce the theorem :

Theorem 2.12. The no-regret control u for the backward heat ill-posed problem (6), is characterized by the unique quadruplet $\{u, \xi, \rho, p\}$ solution to the optimality system :

$$
\mid \begin{aligned}
& y^{\prime}-\Delta y=u, \quad-\xi^{\prime}-\Delta \xi=y, \quad \rho^{\prime}-\Delta \rho=0, \\
& \text { and }-p^{\prime}-\Delta p=y-z_{d}+\rho \quad \text { in } Q, \\
& y(0)=y(T)=0, \quad \xi(T)=0, \quad \text { and } \\
& \rho(0)=\lambda(0), \quad p(T)=0 \quad \text { in } \Omega, \\
& y=0, \quad \xi=0, \quad \rho=0, \quad p=0 \quad \text { on } \Sigma,
\end{aligned}
$$

and the variational inequality :

with $u \in \mathcal{U}_{a d}, y \in L^{2}\left(0, T ; L^{2}(\Omega)\right)$ and

$$
\langle p+N u, v-u\rangle \geq 0 \quad \forall v \in \mathcal{U}_{a d}
$$

$$
p, \rho, \xi \in L^{2}\left(0, T ; H^{2}(\Omega) \cap H_{0}^{1}(\Omega)\right), \quad \lambda \in L^{2}(\Omega) .
$$


Remark 2.13. As we have seen in this work, the hypothesis (12) is replaced by the no-regret notion. This method gives another point of view of solving the control problem of singular distributed systems.

\section{The CONTROLlability APPROACH}

Instead of the regularization for the backward ill-posed approach, we here use the null-controllability approach in Dorville et al. [3] for the regular heat equation (of incomplete data). With the low-regret method, we obtain at the end an optimality system for the backward ill-problem.

Consider the following heat system :

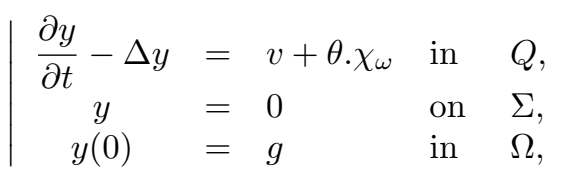

where $v \in L^{2}(Q), \omega \subset \subset \Omega$ an open set, $g \in L^{2}(\Omega)$, and $\chi_{\omega}$ such that

$$
\chi_{\omega} u=\left\{\begin{array}{cc}
u & \text { on } \\
0 & \text { else }
\end{array}(0, T) \times \omega\right.
$$

For every fixed $(v, g, \theta)$ the system (39) admits a unique solution $y=y(v, g, \theta)$. The null controllability problem for (39) consists in finding $\theta \in L^{2}\left(0, T ; L^{2}(\omega)\right)$ such that if $y$ is solution of (39) then

$$
y(T)=0 .
$$

This problem is classical and well-known (see for example Puel [16] and the references therein). Moreover, the solution is not unique.

Suppose selected a unique solution -by a given criterion- for (39)(40). More precisely, we will see that there is a criterion related to a weight function $\rho$ such that for given $(v, g)$, there is a unique pair $\left(\theta, y_{\theta}\right)$ such that

$$
y_{\theta}=y(v, g ; \theta) \quad \text { and } \quad y_{\theta}(T)=0 \text {. }
$$

Denote by $\theta=\theta(v, g)$ and $y_{\theta}=y_{\theta}(v, g)$. We can notice that $y_{\theta}(0,0)=0$ iff $\theta(0,0)=0$. With this, for $(v, g) \in L^{2}(Q) \times L^{2}(Q)$, we consider the cost function

$$
J(v, g)=\left\|y_{\theta}(v, g)-z_{d}\right\|_{L^{2}(Q)}^{2}+N\|v\|_{L^{2}(Q)}^{2}
$$

where $z_{d} \in L^{2}(Q)$ and $N \in \mathbb{R}_{+}^{*}$ are given. Then the goal is to study the optimal control of this controlled system. The question of control for controllability problems of incomplete data is new, and was treated in detail by Dorville [1], and by Dorville et al. [3] very recently. We recall a variational method which ensures the existence and uniqueness selection of a solution for (39)(40).

The following lemma is slightly different (simpler) from lemma 1 in [3].

Lemma 3.1. Denote by $L=\frac{\partial}{\partial t}-\Delta$ and $L^{*}=-\frac{\partial}{\partial t}-\Delta$ the adjoint operator. Then there is a $C^{2}$ weighted positive function $\rho$ on $Q$ such that $\frac{1}{\rho}$ is bounded in $Q$, and there is a constant $C=C(\Omega, T, \omega, \rho)>0$ such that

$$
\int_{Q} \frac{1}{\rho^{2}}|q|^{2} d x d t+\int_{\Omega}|q(0)|^{2} d x \leq C\left[\int_{Q}\left|L^{*} q\right|^{2} d x d t+\int_{0}^{T} \int_{\omega} \frac{1}{\rho^{2}}|q|^{2} d x d t\right]
$$


for every $q \in \mathcal{V}=\left\{\varphi \in C^{\infty}([0, T] \times \bar{\Omega}) \quad\right.$ tel que $\left.\varphi_{\left.\right|_{\Sigma}}=0\right\}$.

This lemma holds from a Carleman inequality, and can be found in [4], or [15] and [16]. The RHS of (42) leads to use the following inner product :

$$
a(r, s)=\int_{Q} L^{*} r L^{*} s d Q+\int_{0}^{T} \int_{\omega} \frac{1}{\rho^{2}} r s d Q .
$$

Denote by $\|.\|_{a}=\sqrt{a(., .)}$ the associated norm on $\mathcal{V}$, and consider the completion given by the Hilbert space $V$. Finally, let be the linear form $\ell$ on $V$ defined by

$$
\ell(s)=\int_{Q} v s d Q+\int_{\Omega} g s(0) d x
$$

Proposition 3.2. Under the hypothesis that $\|\rho v\|_{L^{2}(Q)}<+\infty$, there is a unique $\widetilde{p} \in V$ solution to :

$$
a(\widetilde{p}, s)=\ell(s) \quad \forall s \in V .
$$

Moreover, if we put

$$
y=L^{*} \widetilde{p}, \quad \text { and } \quad \theta=-\frac{1}{\rho^{2}} \widetilde{p} \cdot \chi_{\omega},
$$

then $\{y, \theta\}$ is the unique solution to (39)(40).

Proof. The proof follows from a simple application of the Lax-Milgram theorem, and by integration by parts.

Remark 3.3. Consider the set $L_{\rho}^{2}(Q)=\left\{w \in L^{2}(Q)\right.$ such that $\left.\rho w \in L^{2}(Q)\right\}$, and denote by $(., . .)_{\rho}=$ $(\rho ., \rho \cdot)_{L^{2}(Q)}$ and $\|\cdot\|_{\rho}=\sqrt{(\cdot, \cdot)_{\rho}}$. From proposition 3.2, for every pair $(v, g)$ with $v \in L_{\rho}^{2}(Q)$ and $g \in L^{2}(\Omega)$, there is a unique pair $\left(\theta, y_{\theta}:=y\right)$ solution of the problem (39)(40). We define the two linear functions

$$
\left\{\begin{array} { c c c } 
{ L ^ { 2 } ( Q ) \times L ^ { 2 } ( \Omega ) } & { \longrightarrow } & { L ^ { 2 } ( 0 , T ; L ^ { 2 } ( \omega ) ) } \\
{ ( v , g ) } & { \longmapsto } & { \theta ( v , g ) }
\end{array} \text { and } \left\{\begin{array}{cl}
L^{2}(Q) \times L^{2}(\Omega) & \longrightarrow L^{2}(Q) \\
(v, g) & \longmapsto y(v, g)
\end{array}\right.\right.
$$

Then $v \longmapsto \theta(v, 0) \in\left(L^{2}\left(0, T ; L^{2}(\omega)\right), \rho d x d t\right)$ and $v \longmapsto y(v, 0) \in L_{\rho}^{2}(Q)$ are continous on $L_{\rho}^{2}(Q)$.

\subsection{The low-regret control for the null-controllable problem}

For every $v \in L_{\rho}^{2}(Q)$, we define the weighted cost function :

$$
J_{\rho}(v, g)=\left\|y(v, g)-z_{d}\right\|_{\rho}^{2}+N\|v\|_{\rho}^{2}
$$

where $z_{d} \in L_{\rho}^{2}(Q)$ and $N>0$. Let be now $\mathcal{U}_{a d}^{\rho}$ a closed convex subset of $L_{\rho}^{2}(Q)$.

By remark 3.3, we have $y(v, g)=y(v, 0)+y(0, g)$ and $\theta(v, g)=\theta(v, 0)+\theta(0, g)$. This, allows to write

$$
J_{\rho}(v, g)-J_{\rho}(0, g)=J_{\rho}(v, 0)-J_{\rho}(0,0)+2(y(v, 0), y(0, g))_{\rho} .
$$

Lemma 3.4. Let be the operator $M: g \longmapsto \widetilde{p}(0, g)$ from $L^{2}(\Omega)$ to $\left(L^{2}(Q), \frac{1}{\rho} d x d t\right)$. Then $M$ is linear continuous on $L^{2}(\Omega)$, and we have

$$
J_{\rho}(v, g)-J_{\rho}(0, g)=J_{\rho}(v, 0)-J_{\rho}(0,0)+2(S(v), g)_{L^{2}(\Omega)}
$$


where $S$ is a linear and continuous operator from $L_{\rho}^{2}(Q)$ to $L^{2}(\Omega)$, such that

$$
S(v)=M^{*}\left(v+\theta(v, 0) \cdot \chi_{\omega}\right)+\widetilde{p}(v, 0)(0),
$$

where $M^{*}$ is the adjoint of $M$.

Proof. The proof is simple and holds from (44) and remark 3.3. Indeed

$$
\begin{aligned}
(y(v, 0), y(0, g))_{\rho} & =\left(v-\frac{1}{\rho^{2}} \widetilde{p}(v, 0) \cdot \chi_{\omega}, \widetilde{p}(0, g)\right)_{L^{2}(Q)}+(\widetilde{p}(v, 0), g)_{L^{2}(\Omega)} \\
& =\left(v-\frac{1}{\rho^{2}} \widetilde{p}(v, 0) \cdot \chi_{\omega}, M g\right)_{L^{2}(Q)}+(\widetilde{p}(v, 0), g)_{L^{2}(\Omega)} .
\end{aligned}
$$

We now give the optimality system of the low-regret control for the problem of missing data (39)-(40) and (46), defined by :

$$
\inf _{v \in \mathcal{U}_{a d}^{\rho}}\left(\sup _{g \in L^{2}(\Omega)}\left(J_{\rho}(v, g)-J_{\rho}(0, g)-\gamma\|g\|_{L^{2}(\Omega)}^{2}\right)\right)
$$

for $\gamma>0$. Using (47), the problem is equivalent to the following classical control problem

$$
\inf _{v \in L_{\rho}^{2}(Q)} \mathcal{J}_{\rho}^{\gamma}(v) \quad \text { where } \quad \mathcal{J}_{\rho}^{\gamma}(v)=J_{\rho}(v, 0)-J_{\rho}(0,0)+\frac{1}{\gamma}\|S(v)\|_{L^{2}(\Omega)}^{2} .
$$

Lemma 3.5. The problem (50) admits a unique solution $u_{\gamma} \in \mathcal{U}_{\text {ad }}^{\rho}$.

Proof. We have the following:

$\mathcal{J}_{\rho}^{\gamma}(v) \geq-J_{\rho}(0,0) \forall v \in \mathcal{U}_{a d}^{\rho}$. Hence $d_{\gamma}=\inf _{v \in \mathcal{U}_{a d}^{\rho}} \mathcal{J}_{\rho}^{\gamma}(v)$ exists. Let then $v_{n}=v_{n}(\gamma)$ be a minimizing subsequence such that $d_{\gamma}=\lim _{n \rightarrow \infty} \mathcal{J}_{\rho}^{\gamma}\left(v_{n}\right)$. We have

$$
-J_{\rho}(0,0) \leq \mathcal{J}_{\rho}^{\gamma}\left(v_{n}\right)=J_{\rho}\left(v_{n}, 0\right)-J_{\rho}(0,0)+\frac{1}{\gamma}\left\|S\left(v_{n}\right)\right\|_{L^{2}(\Omega)}^{2} \leq d_{\gamma}+1 .
$$

We deduce the following estimates :

$$
\left\|v_{n}\right\|_{\rho} \leq c_{\gamma}, \quad \frac{1}{\sqrt{\gamma}}\left\|S\left(v_{n}\right)\right\|_{L^{2}(\Omega)} \leq c_{\gamma}, \quad\left\|y\left(v_{n}, 0\right)-z_{d}\right\|_{\rho} \leq c_{\gamma},
$$

$\left(c_{\gamma}\right.$ independent of $\left.n\right)$. Then, there is $u_{\gamma} \in \mathcal{U}_{a d}^{\rho}$ such that $v_{n} \rightarrow u_{\gamma}$ weak in $L_{\rho}^{2}(Q)$. Also, $y\left(v_{n}, 0\right) \rightarrow y\left(u_{\gamma}, 0\right)$ (continuity with respect to the data). At last, thanks to the convexity of $\mathcal{J}_{\rho}^{\gamma}$, we deduce that $u_{\gamma}$ is unique.

Theorem 3.6. The low-regret control $u_{\gamma} \in \mathcal{U}_{a d}^{\rho}$ is characterized by the quadruplet $\left\{u_{\gamma}, y_{\gamma}, \widetilde{\theta}_{\gamma}, p_{\gamma}\right\} \in \mathcal{U}_{a d}^{\rho} \times$ $L^{2}(Q) \times V \times L^{2}(Q)$ unique solution of the system

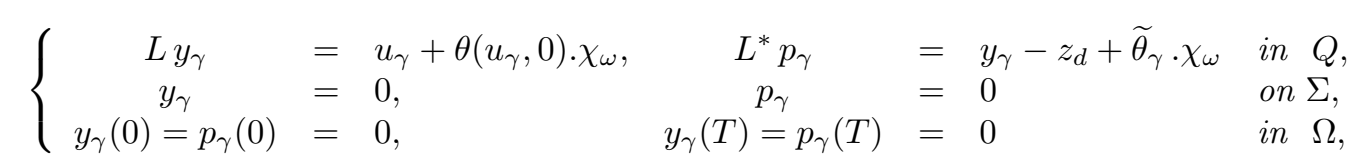

where $y_{\gamma}=y\left(u_{\gamma}, 0\right), p_{\gamma}=p\left(u_{\gamma}, 0\right), \widetilde{\theta}_{\gamma}=\widetilde{\theta}\left(u_{\gamma}, 0\right)$, and the variational inequality

$$
\left(T^{*}\left[L p_{\gamma}-\tilde{\theta}_{\gamma} \cdot \chi_{\omega}\right]+N \rho^{2} u_{\gamma}+(1 / \gamma) S^{*} S\left(u_{\gamma}\right), v-u_{\gamma}\right)_{L^{2}(Q)} \geq 0 \quad \forall v \in \mathcal{U}_{a d}^{\rho}
$$


where $S^{*}$ is the adjoint of $S$, and $T^{*}$ is the adjoint of the operator $T: v \mapsto L^{*} \widetilde{p}(v, 0)$.

Proof. The necessary condition of Euler-Lagrange satisfied by $u_{\gamma}$ gives $\left(y_{\gamma}-z_{d}, \rho^{2} y\left(v-u_{\gamma}, 0\right)\right)_{L^{2}(Q)}+N\left(\rho^{2} u_{\gamma}, v-u_{\gamma}\right)_{L^{2}(Q)}+\frac{1}{\gamma}\left(S\left(u_{\gamma}\right), S\left(v-u_{\gamma}\right)\right)_{L^{2}(\Omega)} \geq 0 \quad \forall v \in \mathcal{U}_{a d}^{\rho}$, $y_{\gamma}=y\left(u_{\gamma}, 0\right)$. We then introduce $\sigma_{\gamma}=\sigma\left(u_{\gamma}, 0\right) \in V$, unique solution of the problem

$$
a\left(\sigma_{\gamma}, q\right)=\int_{Q}\left(y_{\gamma}-z_{d}\right) q d x d t, \quad \forall q \in V
$$

We then define the adjoint state $p_{\gamma}=p\left(u_{\gamma}, 0\right)$ by

$$
p_{\gamma}=L^{*} \sigma_{\gamma} \quad \text { and we put } \quad \tilde{\theta}_{\gamma}=-\frac{1}{\rho^{2}} \sigma_{\gamma}
$$

Then the pair $\left(\widetilde{\theta}_{\gamma}, p_{\gamma}\right)$ is solution of the null-controllability problem :

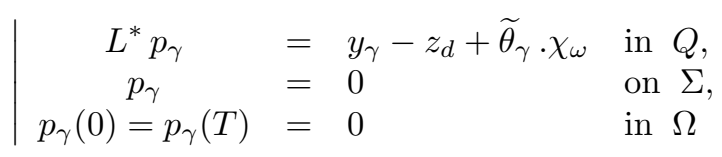

Then the Euler condition writes :

$$
\left(L p_{\gamma}-\tilde{\theta}_{\gamma} \cdot \chi_{\omega}, \rho^{2} y\left(v-u_{\gamma}, 0\right)\right)_{L^{2}(Q)}+\left(N \rho^{2} u_{\gamma}+(1 / \gamma) S^{*} S\left(u_{\gamma}\right), v-u_{\gamma}\right)_{L^{2}(Q)} \geq 0
$$

¿From another side we have

$$
\left(L p_{\gamma}-\tilde{\theta}_{\gamma} \cdot \chi_{\omega}, \rho^{2} y\left(v-u_{\gamma}, 0\right)\right)_{L^{2}(Q)}=\left(T^{*}\left(L p_{\gamma}-\tilde{\theta}_{\gamma} \cdot \chi_{\omega}\right), v-u_{\gamma}\right)_{L^{2}(Q)} \quad \forall v \in \mathcal{U}_{a d}^{\rho}
$$

where $T^{*}$ is the adjoint of the linear continuous operator $T: v \mapsto L^{*} \widetilde{p}(v, 0)$ de $L_{\rho}^{2}(Q)$ in $\left(L_{\rho}^{2}(Q), \rho^{2} d x d t\right)$.

\subsection{Passage to the limit}

In this section, we verify that we also have an optimality for the no-regret control. It remains to see that $y_{\gamma}$ (resp. $p_{\gamma}$ ) particularly satisfies to the well-posed system :

$$
\left.(3 *) \mid \begin{array}{l|l}
L y_{\gamma}=u_{\gamma}+\theta\left(u_{\gamma}, 0\right) \chi_{\omega} \in L^{2}(Q), \\
y_{\gamma}=0, \\
y_{\gamma}(0)=0,
\end{array} \quad \begin{array}{l}
L^{*} p_{\gamma}=y^{\gamma}-z_{d}+\widetilde{\theta}_{\gamma} \chi_{\omega}, \\
p_{\gamma}=0 \text { on } \Sigma, \\
p_{\gamma}(T)=0,
\end{array}\right)
$$

and of course $y_{\gamma}(T)=0$ (resp. $\left.p_{\gamma}(0)=0\right)$.

The system $(3 *)$ implies

$$
\left\|y_{\gamma}\right\|_{L^{2}\left(0, T ; H_{0}^{1}(\Omega)\right)}+\left\|y_{\gamma}^{\prime}\right\|_{L^{2}\left(0, T ; H^{-1}(\Omega)\right)} \leq C,
$$

(resp. $(4 *)$ gives $\left.\left\|p_{\gamma}\right\|_{L^{2}\left(0, T ; H_{0}^{1}(\Omega)\right)}+\left\|p_{\gamma}{ }^{\prime}\right\|_{L^{2}\left(0, T ; H^{-1}(\Omega)\right)} \leq C\right)$.

Then $y_{\gamma} \rightarrow y$ (resp. $p_{\gamma} \rightarrow p$ ) weakly in $L^{2}\left(0, T ; H_{0}^{1}(\Omega)\right.$ ), and by compactness $y_{\gamma} \rightarrow y$ (resp. $p_{\gamma} \rightarrow p$ ) stronly in $L^{2}\left(0, T ; L^{2}(\Omega)\right)$.

Also,

$$
\frac{1}{\gamma} S^{*} S\left(u_{\gamma}\right) \rightarrow S^{*} S(u)
$$


strongly in $L^{2}\left(0, T ; L^{2}(\Omega)\right)$ by the same arguments, since

$$
\left\|S^{*} S(u)\right\|_{L^{2}(\Omega)} \leq \lim _{\gamma \rightarrow 0} \frac{1}{\sqrt{\gamma}}\left\|S^{*} S\left(u_{\gamma}\right)\right\|_{L^{2}(\Omega)} \leq C .
$$

We then obtain the following singular optimality system, stated in the following theorem :

Theorem 3.7. The no-regret control $u \in \mathcal{U}_{\text {ad }}^{\rho}$ is characterized by the quadruplet $\{u, y, \tilde{\theta}, p\} \in \mathcal{U}_{a d}^{\rho} \times L^{2}(Q) \times$ $V \times L^{2}(Q)$, unique solution of the system

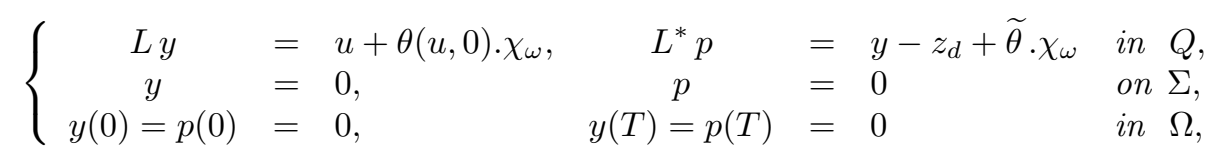

where $y=y(u, 0), p=p(u, 0), \widetilde{\theta}=\widetilde{\theta}(u, 0)$,

with the variational inequality

$$
\left(T^{*}\left[L p-\widetilde{\theta} \cdot \chi_{\omega}\right]+N \rho^{2} u+S^{*} S(u), v-u\right)_{L^{2}(Q)} \geq 0 \quad \forall v \in \mathcal{U}_{a d}^{\rho},
$$

where $S^{*}$ is the adjoint of $S$, and $T^{*}$ the adjoint of the operator $T: v \mapsto L^{*} \widetilde{p}(v, 0)$.

Remark 3.8. As a general remark on this section, we see that the optimality system has the same structure as the one of standard optimal control problems. More precisely, the adjoint state satisfies to a null-controllability system, as for the state $y$.

\section{REFERENCES}

[1] Dorville R. Sur le contrôle de quelques problèmes singuliers associés à l'équation de la chaleur (On the control of some singular problems associated to the heat equation). Thèse de Doctorat de l'Université des Antilles et de la Guyane, Guadeloupe (2004).

[2] Dorville R., Nakoulima O., Omrane A. Low-regret control for singular distributed systems: The backwards heat ill-posed problem. Applied Math. Letters, 17/5, pp 549-552 (2004).

[3] Dorville R., Nakoulima O., Omrane A. Contrôle optimal pour les problèmes de contrôlabilité de systèmes distribués à données manquantes. C. R. Acad. Sci. Paris Ser. I Math., Vol. 338, pp 921-924 (2004).

[4] Emanuvilov O. Yu. Controllability of Parabolic Equations. Sbornik Mathematics, Vol. 186, No 6, pp 879-900 (1995).

[5] Lions J. L. Contrôle optimal des systèmes gouvernés par des équations aux dérivées partielles. Dunod, Paris (1969).

[6] Lions J. L. Contrôle optimal pour les systèmes distribués singuliers. Gauthiers-Villard, Paris (1983) .

[7] Lions J. L. Perturbations singulières dans les problèmes aux limites et en contrôle optimal. Lecture Notes in Mathematics, Springer (1973).

[8] Lions J. L. Contrôle à moindres regrets des systèmes distribués. C. R. Acad. Sci. Paris Ser. I Math., Vol. 315, pp 1253-1257 (1992).

[9] Lions J. L. No-regret and low-regret control, Environment, Economics and Their Mathematical Models, Masson, Paris, 1994.

[10] Lions J. L. Duality Arguments for Multi Agents Least-Regret Control. Collège de France, Paris (1999).

[11] Lions J.-L., Magenes E. Problèmes aux limites non homogènes (I) et (II), Ann. Sc. Norm. Sup. Pisa, Vol. 14, pp 259-308, et Vol. 15, (1962), pp 39-101 (1961) .

[12] Lions J.-L., Magenes E. Remarques sur les problèmes aux limites linéaires elliptiques, Rend. Acc. Naz. Lincei, Vol. 8/32, pp 873-883 (1962).

[13] Nakoulima O., Omrane A., Velin J. Perturbations à moindres regrets dans les systèmes distribués à données manquantes. C. R. Acad. Sci. Paris Ser. I Math., Vol. 330, pp 801-806 (2000).

[14] Nakoulima O., Omrane A., Velin J. On the pareto control and no-regret control for distributed systems with incomplete data. SIAM J. Control Optim. vol. 42, N 4, pp. 1167-1184 (2003).

[15] Puel J. P. Controlabilité exacte et approchée. Cours de DEA, Université de Paris 6 (2000).

[16] Puel J. P. Applications of global Carleman inequalities to controllability and inverse problems. (to appear)

[17] Savage L. J. The Foundations of Statistics. Dover, 2nd Edition (1972). 